\title{
Autism and Inner Speech: A Computational Model of Language Functions in Autistic Flexible Behaviour
}

Giovanni Granato ( $\sim$ giovanni.granato@istc.cnr.it)

Institute of Cognitive Sciences and Technologies

Anna M. Borghi

Department of Dynamic and Clinical Psychology, and Health Studies

Andrea Mattera

Institute of Cognitive Sciences and Technologies

Gianluca Baldassarre

Institute of Cognitive Sciences and Technologies

\section{Research Article}

Keywords: Inner speech, Autism Spectrum Condition, Cognitive flexibility, Ageing, WCST, Computational model

Posted Date: August 2nd, 2021

DOl: https://doi.org/10.21203/rs.3.rs-744730/v1

License: (9) This work is licensed under a Creative Commons Attribution 4.0 International License. Read Full License 


\title{
Autism And InNer Speech: A Computational Model of LANGUAGE FunCtions IN Autistic FleXible BeHAVIOUR
}

\author{
Giovanni Granato* \\ Laboratory of Computational Embodied Neuroscience \\ Institute of Cognitive Sciences and Technologies \\ National Research Council of Italy, \\ Rome, Italy \\ School of Computing, Electronics and Mathematics \\ University of Plymouth \\ Plymouth, U.K. \\ giovanni.granato@istc.cnr.it \\ Andrea Mattera \\ Laboratory of Computational Embodied Neuroscience \\ Institute of Cognitive Sciences and Technologies \\ National Research Council of Italy \\ Rome, Italy \\ andrea.mattera@istc.cnr.it
}

\author{
Anna M. Borghi \\ Dipartimento di Psicologia Dinamica, Clinica e Salute \\ Sapienza University of Rome \\ Institute of Cognitive Sciences and Technologies \\ National Research Council of Italy \\ Rome, Italy \\ anna. borghi@uniroma1. it \\ Gianluca Baldassarre \\ Laboratory of Computational Embodied Neuroscience \\ Institute of Cognitive Sciences and Technologies \\ National Research Council of Italy \\ Rome, Italy \\ gianluca.baldassarre@istc.cnr.it
}

July 23, 2021

\begin{abstract}
Experimental and computational studies propose that inner speech boosts categorisation skills and executive functions, making human behaviour more focused and flexible. In addition, many clinical studies highlight a relationship between poor inner-speech and an executive impairment in autism spectrum condition (ASC), but contrasting findings are reported. Here we investigate the latter issue through a previously implemented and validated computational model of the Wisconsin Cards Sorting Tests. In particular, the model was applied to detect the individual differences in cognitive flexibility and inner speech contribution in ASC and neurotypical participants. Our results suggest that the use of inner-speech increases along the life-span of neurotypical participants but is absent in ASC ones. Although we found more attentional failures in autistic children/teenagers and more perseverative behaviours in autistic young/older adults, only ASC children and ASC older adults exhibited a lower performance than matched control groups. Overall, our results corroborate the idea that the lower use of inner speech in ASC teenagers and young adults is compensated by alternative cognitive strategies (e.g., visual thinking), but it could represent a disadvantage for children (for the missing support of development) and older adults (for the missing compensation of cognitive decline). Moreover, the results suggest that cognitive-behavioural therapies should focus on developing inner speech skills in ASC children as this could provide cognitive support along their whole life span.
\end{abstract}

Keywords Inner speech $\cdot$ Autism Spectrum Condition $\cdot$ Cognitive flexibility $\cdot$ Ageing $\cdot$ WCST $\cdot$ Computational model

\section{Introduction}

Humans commonly use inner speech, a covert and self-directed form of language without motor articulation [1]. Evidence has shown that inner speech plays an important role in supporting cognition. In particular, it boosts 
categorisation processes [2, 3], executive functions [4, 5, 6], working memory [7], metacognition [8], and motivation [9].

Several experimental and clinical studies started to investigate the role of inner speech in psychiatric and neurodiversity conditions (for a recent review, see [10]). These studies show that inner speech can generally provide cognitive support, but in some cases it can also have disruptive effects. For example, in schizophrenic patients it can be distracting, fragmented, charged with negative emotions, and possibly involve auditory hallucinations.

Here we focus on the Autism Spectrum Condition (ASC) and, in particular, on mild autism, that is, a neurodiversity condition that leads to repetitive behaviours, social impairment, sensory alterations, and restricted interests [11]. 'Mild autism' is a diagnostic label, previously corresponding to the 'Asperger syndrome'/'high-functioning autism', that involves low intensity symptoms compared to the other two severity levels ('moderate' and 'severe'). There is also an open debate regarding the use of the terms 'condition' or 'disorder' to refer to mild autism/Asperger Syndrome/highfunctioning autism [12]. Here we use the term 'condition' to avoid stigma without ignoring the daily challenges and possible impairments it involves.

Many studies investigated the relationship between inner speech and executive functions in ASC, and contrasting results are reported (for a review see [13]). For example, some studies on planning [14, 15, 16] found that an experimental interference of inner speech (e.g., articulatory suppression) impairs planning abilities in control participants but not in an ASC population. However, the results should be taken with caution due to potential methodological limitations (see critiques by [13]). Again, evidence on working memory suggests that ASC individuals do not spontaneously use inner speech to name stimuli internally (e.g., [17]) while most studies on motor control indicate either that ASC individuals use inner speech or that the absence of inner speech does not impact their performance. Crucially for us, [18] showed that articulatory suppression does not interfere with cognitive flexibility in ASC people, who do not show an impaired performance. However, [19] found that ASC children performed worse than controls even if they did used private speech. Overall, these scattered and controversial results leave space to further research. In particular, findings suggest that autistic people make a reduced use of inner speech but it is debated whether this reduction has an impact on executive functioning and in particular on cognitive flexibility.

Here we use a computational modelling approach to investigate the relationship between inner speech and executive functions in autistic people. Specifically, we used here a previously implemented and validated computational model [6] able to perform the Wisconsin Cards Sorting test (WCST), a neuropsychological test commonly used to measure cognitive flexibility [20]. The model reproduced several human behavioural data, and was also able to distinguish the different levels of inner speech contribution during an experimental protocol involving verbal shadowing. Here we use the model to address four studies in which the WCST is administered to ASC children [21], teenagers [22], young adults [23] and old adults [24].

Our results suggest that the control groups show an inner speech contribution that progressively increases with the age of the participants. Instead, autistic people do not show an inner speech contribution at any age. Moreover, although we found more attention failures in autistic children/teenagers and more perseveration in autistic young/old adults, only children and old adults show statistically significant lower global performances than their control groups. Therefore we propose that for neurotypical people (not autistic) inner speech is a solid cognitive tool in all ages, representing a development support for children and a compensating tools for old adults (cognitive decline). On the other hand, the absence of inner speech in autism can be compensated in teenagers and young adults (e.g., based on above-average visual skills and thinking; [25, 26, 27]), but can represent an impairing factor for children and old adults.

The presented results have clinical implications. In particular, they suggest focusing psychotherapies treatments on the development of inner speech skills, especially in ASC children. The suitable integration of inner speech and strong visual thinking abilities could indeed be a protecting factor against ageing of autistic people.

\section{Methods}

\subsection{Task and participants data}

The WCST [28] is a neuropsychological test that is commonly used to measure cognitive flexibility, the capacity to change behavioural strategies to achieve a target goal on the basis of external feedback [20]. The task setting is composed of two decks of 64 cards and four target cards, both put on a table in front of the participant (Figure 1). Each deck card shows a specific combination of elements varying with respect to three categories (colour, shape, number), each characterised by four attributes (colour: red, green, blue, yellow; shape: stars, triangles, circles, crosses; number: one, two, three, four). Each target card shows a unique combination of attributes (e.g. one red triangle, two green circles, etc.). In our simulations the third category (numerosity) is substituted with size (small, medium small, medium large, and large), and the star and cross shapes are substituted with square and bar shapes to reduce the resolution 
of the images and gain simulation speed. These modifications do not alter the quality of the results. Participants are required to sort each deck-card, choosing one of the three categories, and put the drawn deck card under one target-card matching it for the attribute of the chosen category. For example, if the chosen category is 'colour', a card with a blue item has to be put under the target card with a blue item. Importantly, there is a correct sorting rule for each turn, but it is unknown to the participant. After each sorting attempt, an external operator provides feedback ('correct' or 'not correct') depending on the current sorting rule and the action executed. The key challenge of the task is hence that the participant has to infer the rule based on the feedback. After a succession of ten correct matches, the sorting rule is changed without informing the participant, who has thus to change the sorting rule to the new one by inferring it on the basis of the feedback.

To extract a complete cognitive profile of participants, we considered four behavioural indices scored during the task performance: Completed Categories (CCs), a global performance index indicating the number of the performed non-interrupted ten-card sequences of correct sorting; Perseverative Errors (PEs), indicating a perseverative behaviour; Non Perseverative Errors (NPEs), indicating an attentional failure or incorrect inferential reasoning; Failure to Maintain Set (FMS), indicating a sustained attention failure.

To investigate the relationship between the inner speech and executive functions in ASC we considered four specific works that (a) adopted the Heaton's version of WCST, (b) involved an ASC group (mild autism/Asperger syndrome/highfunctioning autism) and a matched control group, and (c) reported at least CC, PE, and NPE indices.

The first group [21] involved 26 children (6 to 12 years) with a diagnosis of autism without mental retardation (DSM-III) and a control group of 52 children matched for age. The second group [22] involved 13 teenagers (16.40 \pm 2.84$)$ with a diagnosis of Asperger syndrome or High-functioning Autism (ICD-10) and a control group of 13 teenagers matched for age and QI. The third group [23] involves 9 young adults $(27 \pm 7)$ with a diagnosis of autism without mental retardation and high verbal competences (DSM-III) and a control group of 10 young adults matched for age, education and QI. The fourth group [24] involves 27 old adults (33.5 \pm 12 ) with a diagnosis of Asperger syndrome (ICD-10) and a control group of 20 old adults matched for age and QI.

Despite the populations of [23] and [24] show similar ages (27 \pm 7 vs $33.5 \pm 12)$ we define them 'young adults' and 'old adults' to better distinguish them. We did not found studies that administrate the WCST to ASC adults older than such age.

\subsection{Model}

A previous version of the model, without an inner speech component, demonstrated to reproduce the behavioural differences between young adults, old adults, frontal patients, and Parkinson patients in the WCST [5]. The model is based on an hypothesis that proposes that flexible goal-directed behaviour is based on the manipulation of perceptual representations, based on internal attention, and the external world, for example to displace objects. In particular, the synergistic integration of internal manipulation of representations and external manipulations of objects allows an agent to support goal-directed flexible perception and behaviour. The hypothesis, and the derived computational model, is based on three main components: a hierarchical visual system, a working memory, and a top-down selector of internal representations.

An updated version of the model was enhanced with the addition of an inner speech component and was validated in [6]. The model reproduced a complete behavioural profile (WCST indices) of three groups of teenagers in different experimental conditions: control, motor tapping and verbal tapping. In particular, it was able to detect the different levels of inner speech contribution of the participants in the control condition and in the verbal shadowing condition.

We present here a description of the model components that allows the reader to interpret the presented results. These components support the cognitive functions needed to perform the task in a goal-directed manner. We reported the computational details of the model in Supplementary Materials and a throughout analysis of the model computational architecture and dynamics is presented in [6].

A first group of components, supporting the internal manipulations of perceptual representations, is as follows. (a) A hierarchical perceptual component that extracts the input visual features at increasing levels of abstraction; this component is analogous to the visual brain system [29], and in the model is implemented as a deep neural-network generative model. (b) A working-memory component that stores the 'priorities' of the task sub-goals (sorting rules), thus determining the probability with which the rules are selected; in the brain, this is a function that is mostly supported by the reentrant circuits of frontal cortices [30, 20] and it is implemented here as a recurrent neural network. (c) A motivational component, using the external feedback to update the information in working memory; in the brain, this function is mostly supported by ventral basal ganglia [31, 32] and it is implemented here as a reinforcement learning (RL) algorithm. (d) A selector and a manipulator, the former that chooses the sorting rule to follow and the latter 
that implement the manipulation of the internal representations by biasing the perceptual system; these functions reflect the top-down control that the fronto-parietal cortex and basal ganglia exert on the perceptual cortices [33, 34]; these functions are implemented here as a softmax function selecting the rule to follow on the basis of the working memory activation, and a mechanism that uses the selected rule to dis-inhibit the high-level representations within the hierarchical perceptual component. (e) An inner-speech component storing a linguistic code on the relevance of the rules to follow, and influencing the working memory rule selections; this component is inspired by the brain systems that integrate linguistic and emotional information [35, 36], and in the model it involves a deep neural-network that produces an output formed by the positive/negative valence, and intensity, of the change of the rule priority.

The model is also formed by additional components that implement a set of sensorimotor auxiliary functions needed to accomplish the WCST and that work as follows. (f) A visual sensor component, extracting the visual information from deck cards and target cards; this component is analogous to the eye retina and in the model it is implemented as an RGB matrix of pixels. (g) A visual comparator component, executing a visual matching of the deck and selected target card based on the comparison of the low-level perceptual representations of the cards reconstructed by the perceptual component under the bias of the chosen rule [5]; in the brain, these processes might rely on an integrated network involving the frontal and temporal-occipital cortices [37, 38], and are implemented here as the computation of the Euclidean distance between the representations of the two cards; (g) A motor component, controlling the saccades on the deck and target cards, and the actions to move the deck card close to the chosen target card after a successful visual matching. After each sorting attempt, a simulated 'external operator', knowing the correct sorting rule, receives the deck card and the chosen target card and returns positive or negative feedback to the model.

The model has four key parameters that influence its computations and behaviour: 'error sensitivity' $(\mu)$, representing the magnitude with which the motivational component influences the working-memory sorting-rule priorities in case of negative feedback; 'memory refresh/forgetting speed' $(\phi)$, representing the decay speed of the working memory rule priorities towards a baseline; 'distractibility/explorative tendency' $(\tau)$, representing the level of randomness of the rule selection; 'inner speech contribution' $(\lambda)$, representing the magnitude with which the inner-speech component influences the working memory. The last parameter is the most important for this study.

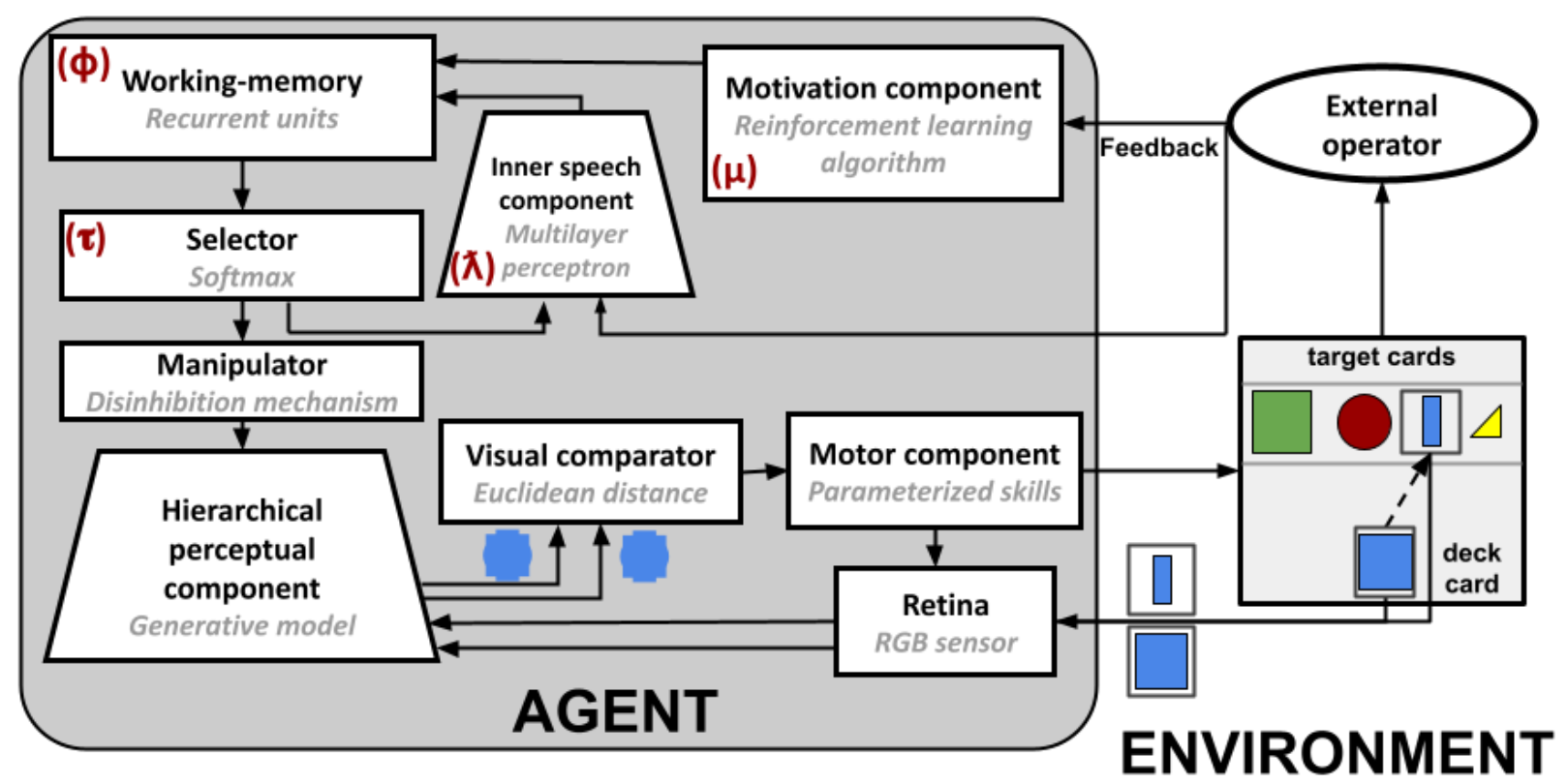

Figure 1: Architecture of the model. In each box, the component label is highlighted in bold and the computational algorithm implemented by the component is highlighted in italic grey. The Greek symbols in red indicate the four key parameters of the model. The two blue little images under the visual comparator highlight that the visual comparison is based on the low-level representations of the deck and selected target cards. These visual representations are produced by the hierarchical component, through its generative capabilities, on the basis of the selected-rule top-down bias (here 'colour'). 


\section{Results}

\subsection{Configurations of parameters of the best fitting models}

As done in [6], we used a statistical search method based on the minimisation of the mean square error (MSE) to find the models parameters (see Supplementary Materials for further details). In particular, this method was used to find the parameter configurations that best reproduced the behavioural data of the control and ASC populations. Although the sample size of some groups was small, the model reproduces the human behavioural data with a low average MSE for both control and ASC groups (see Table S1 in Supplementary Materials).

Table 1 and Figure 2 show the parameter values of the models populations that best fit the dataset of the human groups. The parameters represent the the simulated cognitive traits of the model and, therefore, of the modelled human participants.

\begin{tabular}{|c|c|c|c|c|}
\hline & $\begin{array}{c}\text { Error } \\
\text { sensitivity } \\
(\mu)\end{array}$ & $\begin{array}{c}\text { Memory refresh, } \\
\text { Forgetting speed } \\
(\phi)\end{array}$ & $\begin{array}{c}\text { Distractibility, } \\
\text { Explorative behaviour } \\
(\tau)\end{array}$ & $\begin{array}{c}\text { Inner speech } \\
\text { contribution } \\
(\lambda)\end{array}$ \\
\hline Control models & & & & \\
\hline Children & 0.08 & 0.37 & 0.18 & 0.17 \\
\hline Teenagers & 0.17 & 0.09 & 0.12 & 0.23 \\
\hline Young adults & 0.21 & 0.73 & 0.12 & 0.33 \\
\hline Old adults & 0.05 & 0.41 & 0.18 & 0.52 \\
\hline ASC models & & & & 0.01 \\
\hline Children & 0.11 & 0.93 & 0.83 & 0.0 \\
\hline Teenagers & 0.20 & 0.19 & 0.14 & 0.02 \\
\hline Young adults & 0.08 & 0.11 & 0.08 & 0.0 \\
\hline Old adults & 0.20 & 0.19 & 0.14 & \\
\hline
\end{tabular}

Table 1: Values of the parameters of the models that produce the best fit of the data on the WCST indices.

Regarding the inner speech contribution (parameter $\lambda$ ), the control groups show an increasing tendency depending on ageing. Differently, ASC groups show an absent or negligible inner speech contribution in all ages.

Regarding the error sensitivity (parameter $\mu$ ), the control groups show an "inverse U-shaped" curve. In particular, children and old adults show a similar and lower error sensitivity, while teenagers and young adults show a similar and higher error sensitivity. In the case of ASC groups, we found similarities among pairs of different groups. In particular, children and young adults show a similar and lower error sensitivity, while teenagers and old adults show the same higher error sensitivity.

Regarding the memory refresh/forgetting speed (parameter $\phi$ ), the control groups again show similarities between children and old adults. Differently, teenagers show the lowest value and young adults the highest value. In case of ASC groups, we found a descending tendency. In particular, children show the highest value with respect to the other groups, and the latter ones show similar values.

Regarding the distractibility/exploratory behaviour (parameter $\tau$ ), the control groups have similar values. Despite this, children and old adults show the same slightly higher value with respect to teenagers and young adults, that show the same value. In the case of ASC groups, similarly to the $\phi$ parameter, we found a descendent tendency. In particular, children show higher value with respect to the other groups, and the latter ones are similar between them.

\subsection{Behavioural comparisons}

\subsubsection{Comparisons between perseverative errors and non perseverative errors in each group}

Since perseverative errors and non perseverative errors identify two opposite tendencies, respectively for perseveration and for distraction [5], we performed statistical comparisons (t-tests with Bonferroni's correction) between PEs and NPEs of each model to investigate its behavioural profile (Figure 3).

The results show that in the control condition only old adults have significant differences in their behavioural profile, with an imbalance toward NPE $(7.9 \pm 2.32 \mathrm{vs} 12.05 \pm 3.53, p<.001)$. In the ASC condition, we found that children have an imbalance toward NPE $(24.77 \pm 4.48$ vs $38.04 \pm 4.4, p<.001)$ while young adults have an imbalance toward 


\section{Parameters of models: trends}

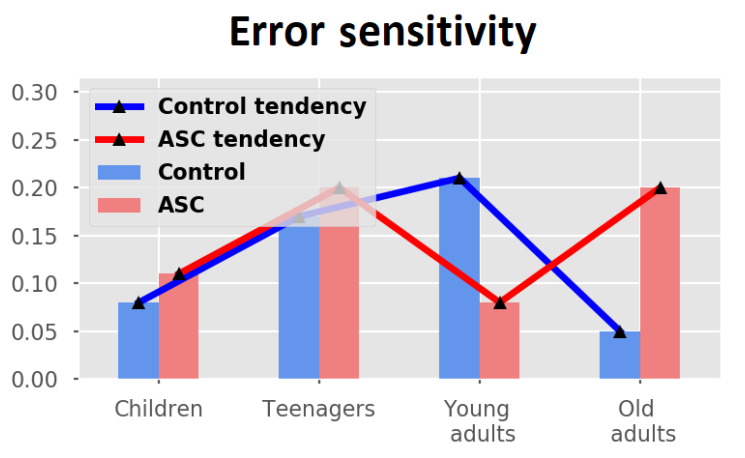

Distractibility, Explorative behaviour

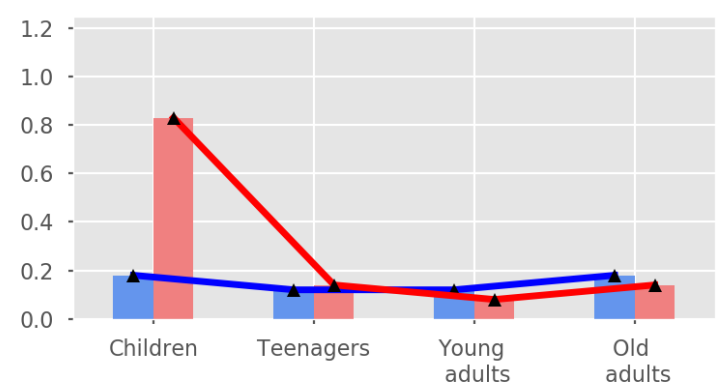

\section{Memory refresh, Forgetting speed}

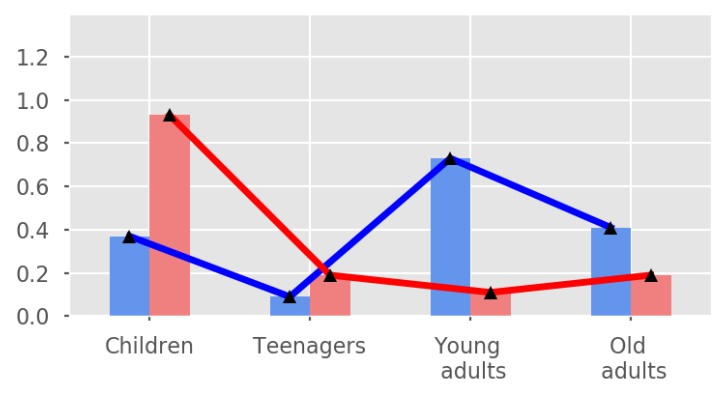

Inner speech contribution

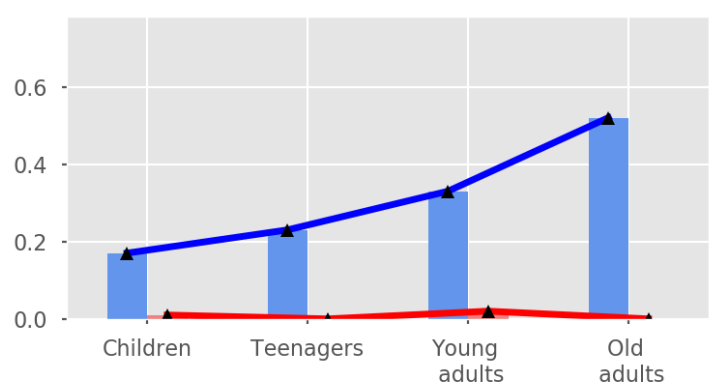

Figure 2: Graphic visualisation of the parameters of the models that best fit the datasets of the human groups (Children, Teenagers, Young adults, Old adults).

Behavioural indices of models (intra-condition analysis: PE vs. NPE)
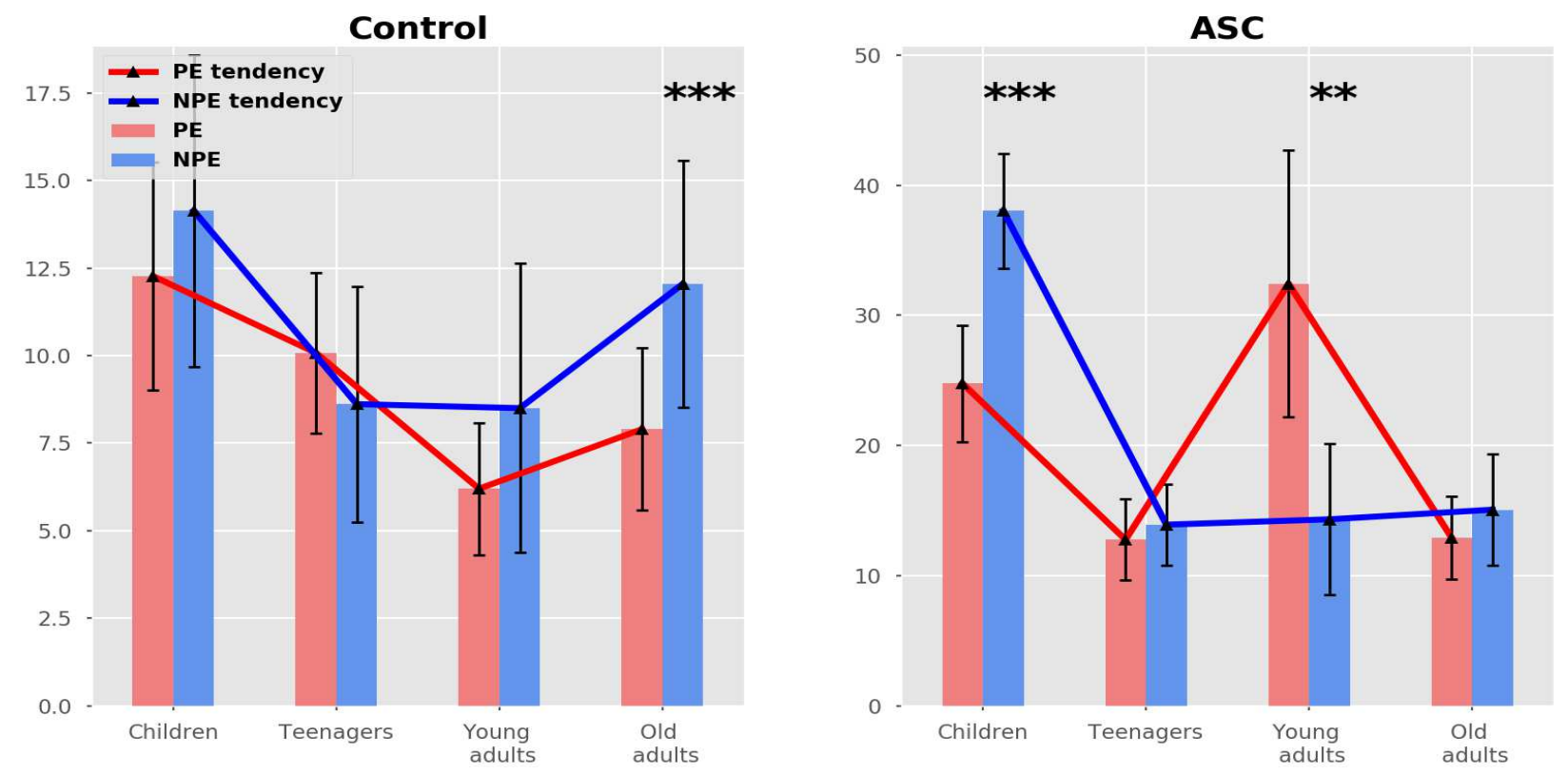

Figure 3: Comparisons between PE and NPE in the control and ASC conditions (Children, Teenagers, Young adults, Old adults). 
PE $(32.44 \pm 10.23$ vs $14.33 \pm 5.79, p<.01)$. Despite the plots show many imbalances of PE and NPE population means in the other groups of models, they also show a high population variability that prevents further statistical differences.

\subsubsection{Comparison between the behaviour of different age groups (intra-condition analysis)}

We performed statistical comparisons (one-way Anova and post-hoc t-tests with Bonferroni's correction) between the models of each condition. These analyses aimed to investigate the differences in the ageing process of control and ASC conditions (Figure 4 , blue and red trend lines).

\section{Behavioural indices of models (inter-conditions analysis: control vs. ASC)}

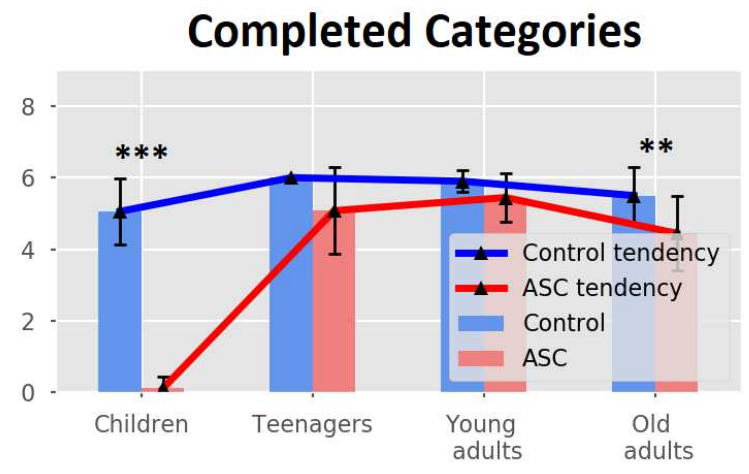

Non Perseverative Errors

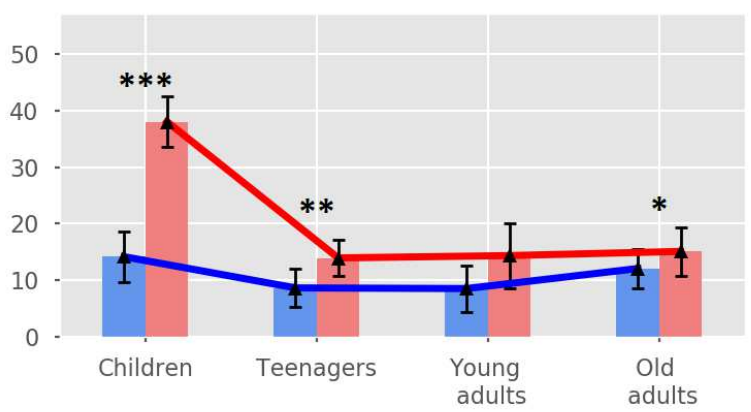

Perseverative Errors

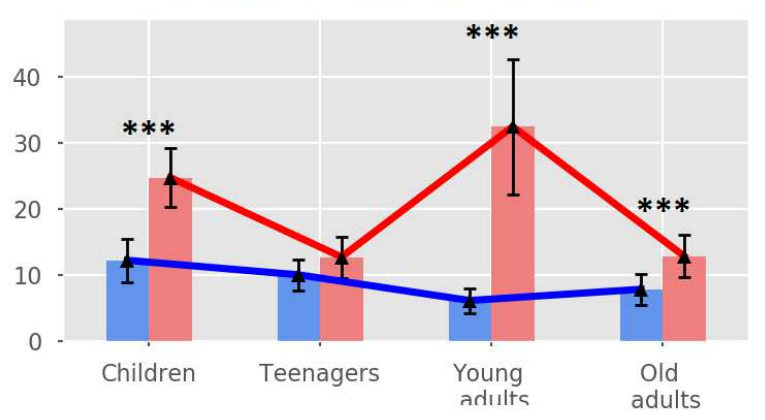

Failures-to-Maintain Sets

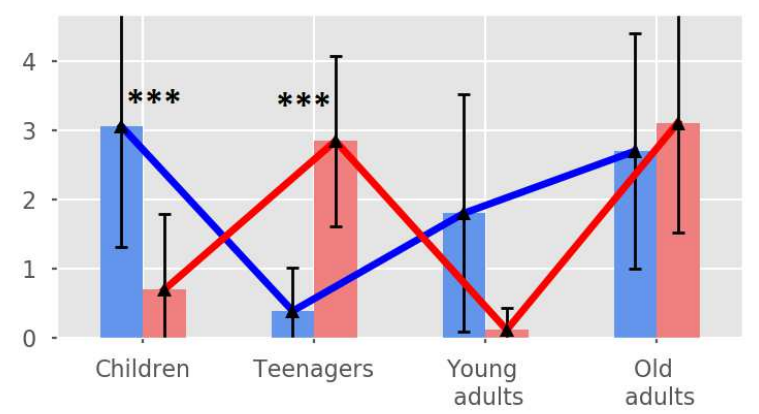

Figure 4: Behavioural indices and comparisons of all models (Children, Teenagers, Young adults, Old adults).

Regarding the completed categories index (CC), we found statistical difference between the control models $(F=7.03$, $p<.001$ ). Post hoc tests (table S2 in supplementary materials) indicate that children achieve a lower CC index with respect to teenagers $(5.06 \pm 0.93$ vs $6.0 \pm 0.0, p<.001)$. We did not find significant statistical differences between the other models, probably due to the high variability of each model population. We found statistical difference between the ASC models $(F>50, p<.001)$. Post hoc tests (Table S3 in Supplementary Materials) indicate that children achieve a low $\mathrm{CC}$ index with respect to teenagers $(0.12 \pm 0.32$ vs $5.08 \pm 1.21, p<.001)$, young adults $(0.12 \pm 0.32$ vs $5.44 \pm 0.68, p<.001)$, and old adults $(0.12 \pm 0.32$ vs $4.44 \pm 1.03, p<.001)$. We did not find further significant statistical differences between the other models.

Regarding perseverative errors (PE), we found statistical difference between the control models $(F=19.87, p<.001)$. Post hoc tests (Table S4 in Supplementary Materials) indicate that children have high PE with respect to young adults $(12.27 \pm 3.26$ vs $6.2 \pm 1.89, p<.001)$ and old adults $(12.27 \pm 3.26$ vs $7.9 \pm 2.32, p<.001)$. We did not find further significant statistical differences between the other models. We found statistical difference between ASC models $(F>50, p<.001)$. Post hoc tests of ASC models (Table S5 in Supplementary Materials) indicate that children show higher PE with respect to teenagers $(24.77 \pm 4.48$ vs $12.77 \pm 3.12, p<.001)$ and old adults $(24.77 \pm 4.48$ vs $12.93 \pm 3.17, p<.001)$, and lower PE with respect to young adults $(24.77 \pm 4.48$ vs $32.44 \pm 10.23, p<.001)$. We did not find further significant statistical differences between teenagers and young adults. 
Regarding non perseverative errors (NPE), we found statistical difference between control models $(F=9.82, p<.001)$. Post hoc tests (Table S6 in Supplementary Materials) indicate that children have high NPE with respect to teenagers $(14.13 \pm 4.44$ vs $8.62 \pm 3.36, p<.001)$ and young adults $(14.13 \pm 4.44$ vs $8.5 \pm 4.13, p<.01)$. We did not find further significant statistical differences between the other models. We found statistical difference between ASC models $(F>50, p<.001)$. Post hoc tests of ASC models (Table S7 in Supplementary Materials) indicate that children have higher NPE with respect to teenagers $(38.04 \pm 4.4$ vs $13.92 \pm 3.12, p<.001)$, young adults $(38.04 \pm 4.4$ vs $14.33 \pm 5.79, p<.001)$, and old adults $(38.04 \pm 4.4$ vs $15.07 \pm 4.29, p<.001)$. We did not find significant statistical differences between the other models.

Regarding failure-to-maintain sets errors (FMS), we found statistical difference between control models ( $F=10.04$, $p<.001$ ). Post hoc tests (Table S8 in Supplementary Materials) indicate that children have high FMS with respect to teenagers $(3.06 \pm 1.75$ vs $0.38 \pm 0.62, p<.001)$, and old adults have higher FMS with respect to teenagers $(2.7 \pm 1.71$ vs $0.38 \pm 0.62, p<.01)$. We did not find significant statistical differences between the other models. We found statistical difference between ASC models $(F=24.31, p<.001)$. Post hoc tests of ASC models (Table S9 in Supplementary Materials) indicate that children have lower FMS with respect to teenagers $(0.69 \pm 1.1$ vs $2.85 \pm 1.23$, $p<.001)$ and old adults $(0.69 \pm 1.1$ vs $3.11 \pm 1.59, p<.001)$. Moreover, teenagers have higher FMS with respect to young adults $(2.85 \pm 1.23$ vs $0.11 \pm 0.31, p<.001)$, and young adults have lower FMS with respect to old adults $(0.11 \pm 0.31$ vs $3.11 \pm 1.59, p<.001)$. We did not find significant statistical differences between the other models.

\subsubsection{Comparison between the behaviour of the control and experimental groups (inter-condition analysis)}

We performed statistical comparisons (t-tests with Bonferroni's correction) between the indices of the control and ASC models to investigate the behavioural differences between them in each age (figure 4

Regarding the completed categories (CC), we found that they are lower in ASC children (5.06 \pm 0.93 vs $0.12 \pm 0.32$, $p<.001)$ and ASC older adults $(5.5 \pm 0.81$ vs $4.44 \pm 1.03, p<.01)$. We did not find a statistical differences in teenagers ( $6.0 \pm 0.0$ vs $5.08 \pm 1.21, p>.05)$ and young adults ( $5.9 \pm 0.3$ vs $5.44 \pm 0.68, p>.05)$.

Regarding perseverative errors (PE), we found that they are higher in ASC children (12.27 \pm 3.26 vs $24.77 \pm 4.48$, $p<.001)$, ASC young adults $(6.2 \pm 1.89$ vs $32.44 \pm 10.23, p<.001)$, and ASC old adults $(7.9 \pm 2.32$ vs $12.93 \pm 3.17$, $p<.001)$. We did not find any statistical difference in teenagers (10.08 \pm 2.3 vs $12.77 \pm 3.12, p>.05)$.

Regarding non perseverative errors (NPE), we found that they are higher in ASC children (14.13 \pm 4.44 vs $38.04 \pm 4.4$, $p<.001)$ and in ASC teenagers $(8.62 \pm 3.36$ vs $13.92 \pm 3.12, p<.01)$. We did not find any statistical difference in young adults $(8.5 \pm 4.13$ vs $14.33 \pm 5.79, p>.05)$ but we found a slightly higher value in ASC old adults $(12.05 \pm 3.53$ vs $15.07 \pm 4.29, p<.05)$.

Regarding the failure-to-maintain set errors (FMS), we found that these are lower in ASC children (3.06 \pm 1.75 vs $0.69 \pm 1.1, p<.001)$ and higher in ASC teenagers $(0.38 \pm 0.62$ vs $2.85 \pm 1.23, p<.001)$. We did not find any statistical difference in young adults $(1.85 \pm 1.72$ vs $0.11 \pm 0.31, p>.05)$ and old adults $(2.7 \pm 1.71$ vs $3.11 \pm 1.59$, $p>.05)$.

\subsection{Internal functioning comparisons}

We also investigated the internal functioning of the models. Figure 5 shows the internal activation of the working memory units of the models recorded during their task performance. The activation of each unit corresponds to a specific sorting rule to follow and the top-space of each plot of the figure shows the errors that occur during each card response.

In the case of children, the activation of the working-memory units of the control and ASC models appear very different. In particular, the ASC model shows several erratic strategy changes that cause the occurrence of several NPE. Interestingly, despite the model is evidently distracted and does not keep the focus on a specific strategy, few PE are scored. As already shown in [6], a participant with high distractability can choose by chance an already tried strategy thus erroneously appearing perseverative. Here we refer to these errors as 'distraction-related PEs'. At last, also the control model shows a sub-optimal performance, caused by reasoning errors (e.g., see the 65-80 interval of trials) and attention failures (e.g. 3-4, 25-35 interval)

In the case of teenagers, the control model shows a good landscape with some negligible reasoning failures (e.g., 0-5 interval) and perseveration (e.g., 40-45 interval). The ASC model shows several 'sustained attentional failures' (e.g., 10-40 interval) and reasoning errors (e.g. 110-120 interval) that cause many NPE and FMS errors. 
Control
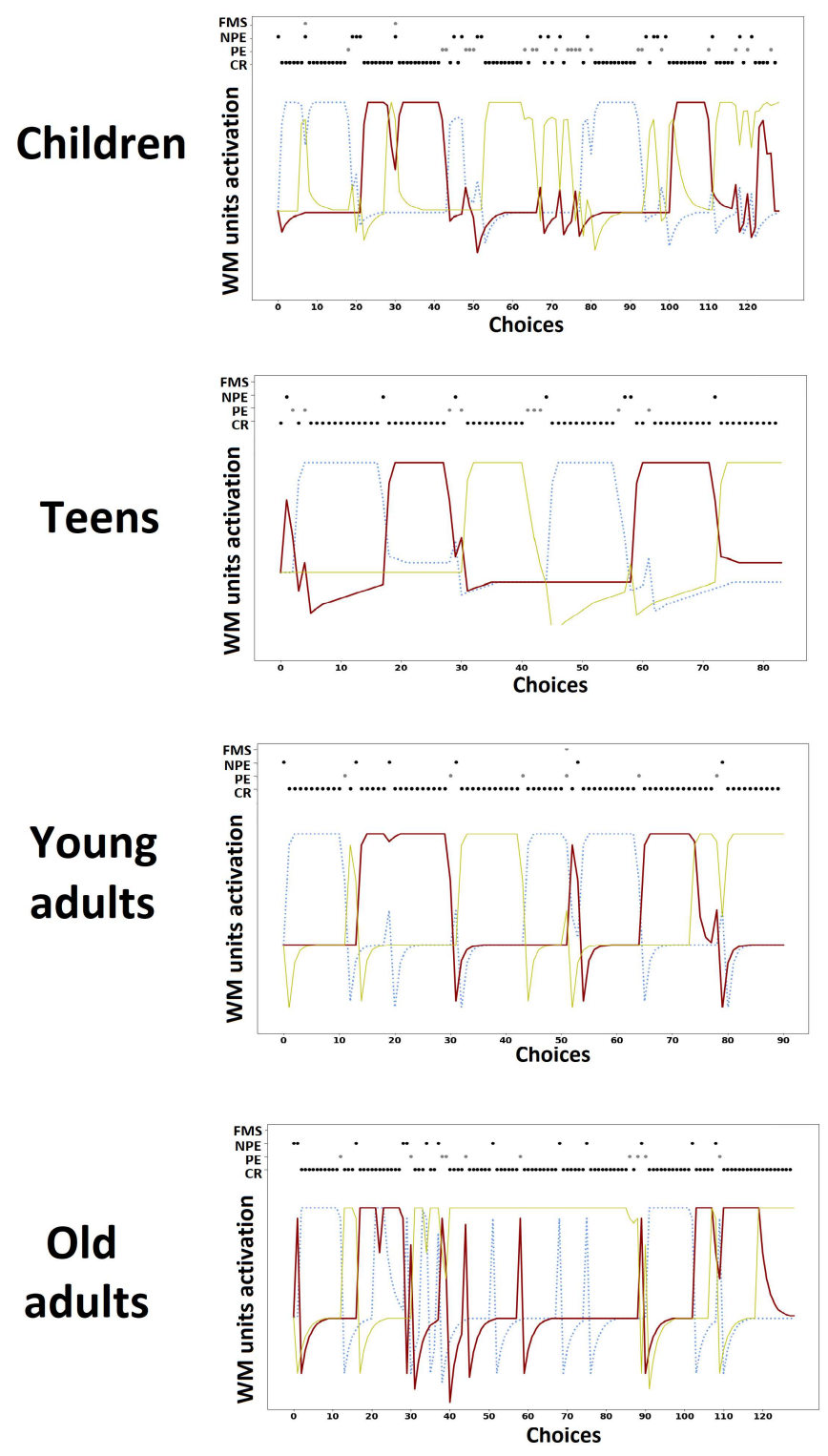

ASC
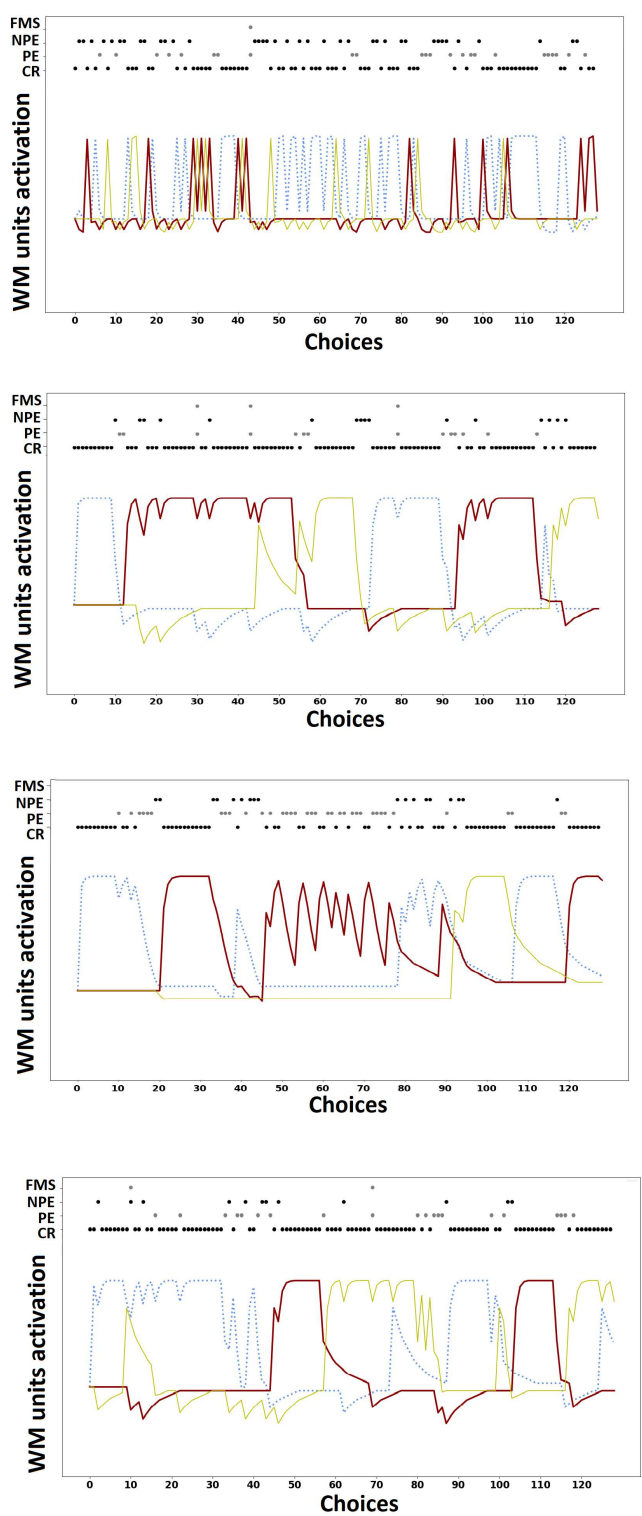

Figure 5: Internal functioning of the executive working memory of the control and ASC models. Each line represents the activation of a memory unit encoding a specific matching rule: thick red line: colour-based matching rule; dotted thin blue line: shape-based matching rule; continuous yellow line: size-based matching rule. The dots at the top of graphs indicate the instances of correct responses (CR) or errors (PE, NPE, FMS).

In the case of young adults, the control model shows a good landscape with minor attention failures (e.g. 15-20, 50-55 intervals). The ASC model shows many perseverative behaviours (e.g. 40-70 interval) and attention failures (e.g. 75-85 interval).

In the case of old adults, both control and ASC models show sub-optimal landscapes. The ASC model shows many attention failures (e.g., 35-45 interval) and reasoning errors (e.g., 55-65 interval), causing many PEs and NPEs. Interestingly, the control model shows many FMS (e.g., 50-75 interval) as the ASC model, showing a poorly focused behaviour. 


\section{Discussion}

The computational model we presented reproduces most behavioural indices of control and ASC humans groups performing the Wisconsin Cards Sorting Test. Moreover, it captures several intra-group and inter-group cognitive and behavioural differences.

Regarding control populations, we generally found similar parameters values between children and old adults (Figure 2 . blue lines) in error sensitivity, memory refresh/forgetting speed, and distractibility/exploratory behaviour, detecting some 'U-shaped tendencies' related to age. Differently from the other three parameters, we found an inner-speech contribution that increases with age, being low in children and high in adults. Further investigations of the cognitive profile of control groups confirmed the $\mathrm{U}$-shaped trends in perseverative errors (perseverative behaviour) and non-perseverative errors (attention/reasoning failures) (Figure 3, left plot). At last, a qualitative analysis of internal activations of the models corroborated these trends (Figure 5), showing that teenagers and young adults exhibit the best performance with respect to children and old adults in which we found more sub-optimal behaviours affected by distraction and perseveration.

Despite the emergence of these trends, the cognitive differences (parameters) between controls groups do not always cause statistically significant differences in behavioural data (Figure 4). For example, only children show significantly lower global performances than the other groups (teenagers, young adults, and old adults), which do not show statistical differences between them.

These results allow the interpretation of contrasting findings on ageing-related effects. In particular, several studies indicate that ageing causes significant brain changes (e.g., [39, 40]), in particular a weakening of executive functions [41, 42, 43], but other studies reveal compensating brain processes such as functional reorganisation and increased bilateral recruitment [44, 45].

Considering this literature, based on the results presented here we suggest that the inner speech contribution, showing an increasing trend from children to old adults, can play an ageing compensation effect. In particular, we propose that inner speech contributes to support early development and to avoid/compensate cognitive decline, thus mitigating the life-span cognitive and behavioural differences between neurotypical individuals. This proposal is also coherent with our results from [6], highlighting that inner speech interacts with the other cognitive processes (working memory storing, error sensitivity, attention), boosting the global performance and diminishing distracted and perseverative behaviours. Moreover, our proposal corroborates the several studies that highlight an important executive modulator function of inner speech in old adults (e.g., [46, 47, 48]).

Regarding the ASC populations, we found relevant differences in cognitive profiles with respect to the control populations. First, we found that ASC groups do not show any contribution of inner speech along the life-span. Second, we found greater differences between children and other groups regarding working memory decay and distractibility. Third, autistic groups show different imbalances with respect to control groups (figure 3, right plot). In particular, autistic children show an evident imbalance toward distractibility (NPE), while young adults show an imbalance toward perseverative behaviours (PE).

These results are particularly interesting because the diagnostic criteria for autism rely on repetitive behaviours [11] and clinical studies mostly focus on perseverative/repetitive behaviours in ASC children and adults [49, 50]. On the other side, several works have investigated attention abnormalities in autism suggesting that an attention impairment could play a causal role in the development of ASC individuals (for a review see [51]). The results presented here agree with these last studies, suggesting that ASC children mostly show an imbalance toward distractions with respect to perseverative behaviours. Moreover, the models suggest a cognitive change in ASC peoples along the life-span, from a distracted profile in children to a perseverative one in young adults.

Regarding behavioural age-related differences, the cognitive traits (parameters) seem to have a more marked effect on behaviours in ASC peoples with respect to the control groups. For example, the descending values of distractibility and memory refresh are reflected by the similar curve of NPE and the low error sensitivity in children and young adults cause higher PE with respect to teenagers and old adults. However, in the case of children this result is evidently altered by many distractibility-related PEs [6]. In particular, the extreme distractibility of ASC children causes a random behaviour (Figure 5. first row) that is sometime scored as 'perseverative behaviour' although it is caused by attention failures (see the imbalance toward NPEs in Figure 3, right plot).

Interestingly, the FMS curve shows a different and unexpected trend with respect to the control trends. In particular, we could expect that ASC children would show higher FMS due high distraction, but in fact they showed a low value of this index. This is probably explained by the difference between NPE and FMS, where the first indicate an attentional/reasoning failure and the second indicates a sustained attention failure. Since ASC children cannot focus on a specific strategy (sorting rule) for long, they often do not achieve the necessary number of responses to occur in a sustained attention error (FMS). These results are coherent with [52], detecting an impairment in selective attention 
and not sustained attention, and with [53], detecting a response inhibition impairment rather than a sustained attention impairment. A high FMS in old adults is another interesting data. While a higher FMS value of teenagers is expected and corroborates a sustained attention deficit [54, 55], we could expect an imbalance toward perseveration in old adults. Instead, we found higher FMS with respect to young adults without a marked PE/NPE imbalance (Figure 3 right plot). These results need further investigations, in particular regarding sustained attention in autistic old adults.

In summary, comparing control and ASC populations we found statistically lower performance only in ASC children and ASC old adults with respect to their control groups. The behaviour comparisons (Figure 4 ) and the analysis of the internal activation of the models (Figure 5] suggests that these differences are caused mainly by more distractions in ASC children/teenagers and a higher perseveration in ASC young/old adults. These results suggest an immature executive functioning in ASC children and a slight cognitive decline in old adults, as suggested by similar trends in the control groups. Despite this, the control groups show weaker intra-condition behavioural differences than the ASC groups, where the difference between age groups appears more marked.

Although many latent variables can contribute to these different behavioural performances (e.g., impaired social learning in autistic children, see [56] for a review), our data suggest that the lack of inner speech development in ASC people could make the ageing effects more evident. In particular, since in control conditions the inner speech represents a cognitive support for an immature executive functioning in children and a compensating process in old adults, its absence in autistic peoples could deprive them of these compensation processes.

Our hypothesis can contribute to explain the contrasting evidence of studies on autism, inner speech and executive functions (for a review see [13]). In particular, the differences might be due to the heterogeneous involved populations that span from children to old adults. Moreover, this proposal is coherent with many studies regarding autism and life-span cognitive changes [57, 58] suggesting that also autistic people show an improvement of executive functions during the life-span. Indeed in ASC teenagers and young adults, compensating processes emerge (e.g., higher visual skills and visual thinking with respect to neurotypical peoples; [26, 27, 25]). However, the lack of inner-speech support still represents a strong impairment for children and old adults.

Finally, our results have interesting clinical implications. Many therapeutic approaches aim to limit compromising symptoms in autism [59], but only few of them focus on speech abilities in autism [60, 61, 62]. These approaches aim to increase linguistic skills to improve social communication abilities, but they do not directly focus on self-directed language (inner-speech). This study suggests that clinicians should device a new class of therapeutic approaches primarily focusing on developing inner speech skills in autistic children. In particular, the integration of early development of inner speech and strong visual thinking could represent an important cognitive support along the life-span of autistic people, from childhood to adulthood.

\section{Conclusions}

This study investigated the relationship between inner speech, ageing, and autism spectrum condition. The study used a previously validated computational model of the Wisonsin sorting cartd test, to reproduce and interpret the data of eight groups of participants differing in age and condition (control condition vs. autism spectrum condition; ASC). The results showed that in the control condition, inner speech contribution increases from childhood to adulthood, possibly supporting early development in children and compensating cognitive decline in old adults. Conversely, the results showed that ASC do not exhibit an inner speech contribution without differences between different ages. Although we found more attention failures in ASC children/teenagers and perseveration in ASC young/old adults, only ASC children and old adults showed lower performances than their matched controls, while no difference was present in teenagers and younger adults. Hence, our results suggest that the absent inner speech support in ASC people creates more difficulties in early development and life-span ageing effects. This hypothesis has clinical implications, suggesting that psycho-therapeutic approaches should focus more on developing inner speech skills in autism spectrum condition.

\section{Authors contribution}

GG: Idea, model design, model implementation, simulations, data analysis, result interpretation, writing. AB: Idea, result interpretation, writing. AM: data analysis, result interpretation, writing. GB: Idea, model design, result interpretation, writing, overall supervision. 


\section{Acknowledgements}

The first author, who is an autistic person, is proud to make this first contribution to a better understanding of the traits of ASC for the benefit of the community.

This research received funding from the European Union's Horizon 2020 Research and Innovation Programme under the project 'GOAL-Robots - Goal-based Open-ended Autonomous Learning Robots', Grant Agreement No 713010.

\section{Competing information}

The authors have no financial, general, or institutional competing interests.

\section{References}

[1] Alderson-Day B, Fernyhough C. Inner speech: development, cognitive functions, phenomenology, and neurobiology. Psychological bulletin. 2015;141(5):931.

[2] Boutonnet B, Lupyan G. Words jump-start vision: A label advantage in object recognition. Journal of Neuroscience. 2015;35(25):9329-9335.

[3] Foerster F, Borghi AM, Goslin J. Labels strengthen motor learning of new tools. Cortex. 2020;129:1 - 10. Available from: http://www.sciencedirect.com/science/article/pii/S0010945220301477

[4] Langland-Hassan P, Vicente A. Inner speech: New voices. Oxford University Press, USA; 2018.

[5] Granato G, Baldassarre G. Internal Manipulation of Perceptual Representations in Human Flexible Cognition: A Computational Model. Neural Networks. 2021.

[6] Granato G, Borghi AM, Baldassarre G. A computational model of language functions in flexible goal-directed behaviour. Scientific reports. 2020;10(1):1-13.

[7] Baddeley A. Working memory. Science. 1992;255(5044):556-559.

[8] Clark A. Magic words: How language augments human computation. Language and thought: Interdisciplinary themes. 1998:162-183.

[9] Morin A. The self-reflective functions of inner speech: Thirteen years later. In: Inner speech: New voices. Oxford University Press, USA; 2018. p. 276-298.

[10] Petrolini V, Jorba M, Vicente A. The role of inner speech in executive functioning tasks: Schizophrenia with auditory verbal hallucinations and autistic spectrum conditions as case studies. Frontiers in Psychology. 2020;11:2452.

[11] Association AP, Association AP, et al. DSM 5. American Psychiatric Association. 2013;70.

[12] Jaarsma P, Welin S. Autism as a natural human variation: Reflections on the claims of the neurodiversity movement. Health care analysis. 2012;20(1):20-30.

[13] Williams DM, Peng C, Wallace GL. Verbal thinking and inner speech use in autism spectrum disorder. Neuropsychology Review. 2016;26(4):394-419.

[14] Wallace GL, Silvers JA, Martin A, Kenworthy LE. Brief report: Further evidence for inner speech deficits in autism spectrum disorders. Journal of autism and developmental disorders. 2009;39(12):1735.

[15] Williams DM, Bowler DM, Jarrold C. Inner speech is used to mediate short-term memory, but not planning, among intellectually high-functioning adults with autism spectrum disorder. Development and psychopathology. 2012;24(1):225-239.

[16] Holland L, Low J. Do children with autism use inner speech and visuospatial resources for the service of executive control? Evidence from suppression in dual tasks. British journal of developmental psychology. 2010;28(2):369-391.

[17] Joseph RM, Steele SD, Meyer E, Tager-Flusberg H. Self-ordered pointing in children with autism: failure to use verbal mediation in the service of working memory? Neuropsychologia. 2005;43(10):1400-1411.

[18] Russell-Smith SN, Comerford BJ, Maybery MT, Whitehouse AJ. Brief report: Further evidence for a link between inner speech limitations and executive function in high-functioning children with autism spectrum disorders. Journal of autism and developmental disorders. 2014;44(5):1236-1243. 
[19] Winsler A, Abar B, Feder MA, Schunn CD, Rubio DA. Private speech and executive functioning among high-functioning children with autistic spectrum disorders. Journal of Autism and Developmental Disorders. 2007;37(9):1617-1635.

[20] Diamond A. Executive functions. Annual review of psychology. 2013;64:135-168.

[21] Shu BC, Lung FW, Tien AY, Chen BC. Executive function deficits in non-retarded autistic children. Autism. 2001;5(2):165-174.

[22] Kaland N, Smith L, Mortensen EL. Brief report: cognitive flexibility and focused attention in children and adolescents with Asperger syndrome or high-functioning autism as measured on the computerized version of the Wisconsin Card Sorting Test. Journal of autism and developmental disorders. 2008;38(6):1161-1165.

[23] Rumsey JM. Conceptual problem-solving in highly verbal, nonretarded autistic men. Journal of autism and developmental disorders. 1985;15(1):23-36.

[24] Ambery FZ, Russell AJ, Perry K, Morris R, Murphy DG. Neuropsychological functioning in adults with Asperger syndrome. Autism. 2006;10(6):551-564.

[25] Mottron L, Dawson M, Soulieres I, Hubert B, Burack J. Enhanced perceptual functioning in autism: an update, and eight principles of autistic perception. Journal of autism and developmental disorders. 2006;36(1):27-43.

[26] Bókkon I, Salari V, Scholkmann F, Dai J, Grass F. Interdisciplinary implications on autism, savantism, Asperger syndrome and the biophysical picture representation: Thinking in pictures. Cognitive Systems Research. 2013;22:67-77.

[27] Grandin T. How does visual thinking work in the mind of a person with autism? A personal account. Philosophical Transactions of the Royal Society B: Biological Sciences. 2009;364(1522):1437-1442.

[28] Heaton R, Chelune G, Talley J, Kay G, Curtiss G, di Hardoy M, et al. WCST: Wisconsin card sorting test : forma completa revisionata : manuale. Firenze: O.S.; 2000.

[29] Konen CS, Kastner S. Two hierarchically organized neural systems for object information in human visual cortex. Nature Neuroscience. 2008;11(2):224-231.

[30] Barraclough DJ, Conroy ML, Lee D. Prefrontal cortex and decision making in a mixed-strategy game. Nature neuroscience. 2004;7(4):404.

[31] Gläscher J, Daw N, Dayan P, O'Doherty JP. States versus rewards: dissociable neural prediction error signals underlying model-based and model-free reinforcement learning. Neuron. 2010;66(4):585-595.

[32] Mannella F, Gurney K, Baldassarre G. The nucleus accumbens as a nexus between values and goals in goal-directed behavior: a review and a new hypothesis. Frontiers in Behavioural Neuroscience. 2013;7:135.

[33] Redgrave P, Prescott TJ, Gurney K. The basal ganglia: a vertebrate solution to the selection problem? Neuroscience. 1999;89(4):1009-1023.

[34] Gazzaley A, Nobre AC. Top-down modulation: bridging selective attention and working memory. Trends in cognitive sciences. 2012;16(2):129-135.

[35] Kotz SA, Meyer M, Paulmann S. Lateralization of emotional prosody in the brain: an overview and synopsis on the impact of study design. Progress in brain research. 2006;156:285-294.

[36] Sidtis JJ, Van Lancker Sidtis D, Dhawan V, Eidelberg D. Switching language modes: complementary brain patterns for formulaic and propositional language. Brain connectivity. 2018;8(3):189-196.

[37] Perani D, Schnur T, Tettamanti M, Cappa SF, Fazio F, et al. Word and picture matching: a PET study of semantic category effects. Neuropsychologia. 1999;37(3):293-306.

[38] Kosslyn SM. Image and brain. 4th ed. Cambridge, MA: The MIT Press; 1999.

[39] Sullivan EV, Adalsteinsson E, Hedehus M, Ju C, Moseley M, Lim KO, et al. Equivalent disruption of regional white matter microstructure in ageing healthy men and women. Neuroreport. 2001;12(1):99-104.

[40] Peters R. Ageing and the brain. Postgraduate medical journal. 2006;82(964):84-88.

[41] Cepeda NJ, Kramer AF, Gonzalez de Sather J. Changes in executive control across the life span: examination of task-switching performance. Developmental psychology. 2001;37(5):715.

[42] Fisk JE, Sharp CA. Age-related impairment in executive functioning: Updating, inhibition, shifting, and access. Journal of clinical and experimental neuropsychology. 2004;26(7):874-890.

[43] Samanez-Larkin GR, Knutson B. Decision making in the ageing brain: changes in affective and motivational circuits. Nature Reviews Neuroscience. 2015;16(5):278-289. 
[44] Cabeza R, Dennis NA. Frontal lobes and aging: deterioration and compensation. Principles of frontal lobe function. 2012;2:628-652.

[45] Daselaar S, Cabeza R, Ochsne K, Kosslyn S. Age-related decline in working memory and episodic memory: Contributions of the prefrontal cortex and medial temporal lobes. The Oxford handbook of cognitive neuroscience. 2013;1:456-472.

[46] Kray J, Eber J, Lindenberger U. Age differences in executive functioning across the lifespan: The role of verbalization in task preparation. Acta Psychologica. 2004;115(2-3):143-165.

[47] Fry PS. Assessment of private and inner speech of older adults in relation to depression. Private speech: From social interaction to self-regulation. 1992:267-284.

[48] John-Steiner V. Private speech among adults. In: Private speech. Psychology Press; 2014. p. 295-306.

[49] Carcani-Rathwell I, Rabe-Hasketh S, Santosh PJ. Repetitive and stereotyped behaviours in pervasive developmental disorders. Journal of Child Psychology and Psychiatry. 2006;47(6):573-581.

[50] Lopez BR, Lincoln AJ, Ozonoff S, Lai Z. Examining the relationship between executive functions and restricted, repetitive symptoms of autistic disorder. Journal of autism and developmental disorders. 2005;35(4):445-460.

[51] Keehn B, Müller RA, Townsend J. Atypical attentional networks and the emergence of autism. Neuroscience \& Biobehavioral Reviews. 2013;37(2):164-183.

[52] Sinzig J, Bruning N, Morsch D, Lehmkuhl G. Attention profiles in autistic children with and without comorbid hyperactivity and attention problems. Acta Neuropsychiatrica. 2008;20(4):207-215.

[53] Johnson KA, Robertson IH, Kelly SP, Silk TJ, Barry E, Dáibhis A, et al. Dissociation in performance of children with ADHD and high-functioning autism on a task of sustained attention. Neuropsychologia. 2007;45(10):22342245.

[54] Christakou A, Murphy C, Chantiluke K, Cubillo A, Smith A, Giampietro V, et al. Disorder-specific functional abnormalities during sustained attention in youth with attention deficit hyperactivity disorder (ADHD) and with autism. Molecular psychiatry. 2013;18(2):236-244.

[55] Murphy CM, Christakou A, Daly EM, Ecker C, Giampietro V, Brammer M, et al. Abnormal functional activation and maturation of fronto-striato-temporal and cerebellar regions during sustained attention in autism spectrum disorder. American Journal of Psychiatry. 2014;171(10):1107-1116.

[56] Tomasello M, Kruger AC, Ratner HH. Cultural learning. Behavioral and brain sciences. 1993;16(3):495-511.

[57] Happé F, Booth R, Charlton R, Hughes C. Executive function deficits in autism spectrum disorders and attentiondeficit/hyperactivity disorder: examining profiles across domains and ages. Brain and cognition. 2006;61(1):25-39.

[58] Pellicano E. The development of core cognitive skills in autism: A 3-year prospective study. Child Development. 2010;81(5):1400-1416.

[59] Aman MG. Treatment planning for patients with autism spectrum disorders. Journal of clinical psychiatry. 2005;66:38.

[60] Adams C, Lockton E, Freed J, Gaile J, Earl G, McBean K, et al. The Social Communication Intervention Project: a randomized controlled trial of the effectiveness of speech and language therapy for school-age children who have pragmatic and social communication problems with or without autism spectrum disorder. International Journal of Language \& Communication Disorders. 2012;47(3):233-244.

[61] Fernandes FDM, Amato CADLH, Molini-Avejonas DR. Language therapy results with children of the autism spectrum. Revista de Logopedia, Foniatría y Audiología. 2012;32(1):2-6.

[62] Flippin M, Hahs-Vaughn DL. Parent couples' participation in speech-language therapy for school-age children with autism spectrum disorder in the United States. Autism. 2020;24(2):321-337. 


\section{Captions of figures and Tables}

Figure 1 - Architecture of the model. In each box, the component label is highlighted in bold and the computational algorithm implemented by the component is highlighted in italic grey. The Greek symbols in red indicate the four key parameters of the model. The two blue little images under the visual comparator highlight that the visual comparison is based on the low-level representations of the deck and selected target cards. These visual representations are produced by the hierarchical component, through its generative capabilities, on the basis of the selected-rule top-down bias (here 'colour').

Table 1 - Values of the parameters of the models that produce the best fit of the data on the WCST indices.

Figure 2 - Graphic visualisation of the parameters of the models that best fit the datasets of the human groups (Children, Teenagers, Young adults, Old adults).

Figure 3 - Comparisons between PE and NPE in the control and ASC conditions (Children, Teenagers, Young adults, Old adults).

Figure 4 - Behavioural indices and comparisons of all models (Children, Teenagers, Young adults, Old adults).

Figure 5 - Internal functioning of the executive working memory of the control and ASC models. Each line represents the activation of a memory unit encoding a specific matching rule: thick red line: colour-based matching rule; dotted thin blue line: shape-based matching rule; continuous yellow line: size-based matching rule. The dots at the top of graphs indicate the instances of correct responses (CR) or errors (PE, NPE, FMS). 


\section{Supplementary Files}

This is a list of supplementary files associated with this preprint. Click to download.

- GranatoetAISupplementaryMaterials.pdf 